\title{
THE LEVEL STRUCTURE OF THE NUCLEUS ${ }^{140} \mathrm{Ce}$
}

\author{
H. W. BAER, J. J. RFIDY and M. L. WIIDEENBECK \\ Department of Physics, University of Michigan, Ann Arbor, Michigan ${ }^{\dagger}$
}

Received 12 April 1966

\begin{abstract}
Precision energy measurements on 24 gamma-ray lines in the ${ }^{1+1} \mathrm{I}$. - -. ${ }^{1+11} \mathrm{Ce}$ decay were performed with a $2 \mathrm{~m}$ curved-crystal spectrometer and a lithium-drifted germanium spectrometer. The relative intensities of 15 ganma rays were determined. A level scheme for ${ }^{1: "} \mathrm{Ce}$ is proposed with the inclusion of two new levels at 2516.14 and $2547.5 \mathrm{keV}$. The new level scheme is compared with recent calculations using the quasi-particle description.
\end{abstract}

\section{Introduction}

The nucleus ${ }^{140} \mathrm{Ce}$ is one of seven stable isotones having a closed neutron shell with $N=82$. It has eight protons outside the $Z=50$ major closed proton shell. In recent years such nuclei have been considered within the framework of the quasiparticle description for spherical nuclei. Some calculations on ${ }^{140} \mathrm{Ce}$ have been performed ${ }^{1,2}$ ). It is thus of interest to determine the energy level structure of ${ }^{140}{ }^{\circ} \mathrm{Ce}$.

Precision measurements of nuclear gamma-ray energies afford a simple check on proposed nuclear energy level structures through the use of the sum rule $E=E_{1}+E_{2}$, where $E_{1}$ and $E_{2}$ are the energies of two cascade transitions and $E$. the corresponding cross-over transition. Since the curved-crystal spectrometer (ces) and lithiumdrifted germanium (Ge(Li)) spectrometer extend considerably the accuracy of many carlier measurements of gamma-ray energies in the ${ }^{140} \mathrm{La} \rightarrow{ }^{140} \mathrm{Ce}$ decaly, the use of this sum rule provides a basis for eliminating some aspects of the previously proposed level schemes, as well as suggesting new possibilities.

A total of 24 gamma-ray energies were measured in the ${ }^{1+0} \mathrm{La}$ decay: 20 were measured with the ces ranging in energy from 64.1 .35 to $2522.6 \mathrm{keV}$; four additional gamma-ray energies ranging in energy from 2348 to $31.23 \mathrm{keV}$ were measured with a $\mathrm{Ge}(\mathrm{Li})$ spectrometer. Using the data from both instruments, the relative intensities of 15 transitions were determined.

The excited levels of ${ }^{140} \mathrm{Ce}$ have been studied by many investigators through the ${ }^{1+0} \mathrm{La} \rightarrow{ }^{140} \mathrm{Ce}$ and ${ }^{1+0} \mathrm{Pr} \rightarrow{ }^{140} \mathrm{Ce}$ decalys. The work before 1960 is compiled in ref. ${ }^{3}$ ) and the monograph by Dzhelepov et al. ${ }^{4}$ ). Later work includes that of Hisatake et al. ${ }^{5}$ ). Takekoshi et al. $\left.{ }^{6}\right)$, Dorikens-Vanpraet ('t al. ${ }^{7}$ ), Black and Mitchell ${ }^{8}$ ), Currie ${ }^{17}$ ) and (iraham and Geiger ${ }^{18}$ ).

+ Work supported in part by L.S. Atomic Energy Commission. 


\section{Experimental Procedures}

\subsection{CURVED-CRYSTAL SPECTROMETER}

The $2 \mathrm{~m}$ curved-crystal spectrometer (ccs) and the source techniques used in this investigation are described in ref. $\left.{ }^{9}\right)$. The measurements were made with the two crystals, $\mathrm{Ge}(022)$ and $\mathrm{Q}(310)$, having effective areas of $4 \mathrm{~cm}^{2}$ and $20 \mathrm{~cm}^{2}$, respectively. Recently the $\mathrm{Ge}(022)$ crystal has been recalibrated using the fourth-through-eighth order reflections of the $411.800 \mathrm{keV}$ transition in the decay of ${ }^{198} \mathrm{Au}$. The resulting calibration constant $7297.989 \pm 0.025 \mathrm{keV} \cdot$ screw-division (s.d.) is slightly smaller than the one previously determined ${ }^{9}$ ). (The calibration constant $k$, the $\gamma$-ray energy $E(\mathrm{keV})$, the crystal position $x$ (s.d.) and the order of reflection are related by $E(x / n)=k$. Also for angles less than $3^{\circ}, 1.0 \mathrm{s.d} . \approx 90 \mathrm{sec}$ of arc.) The calibration constant for the $Q(310)$ crystal is $12369.94 \pm 0.50 \mathrm{keV} \cdot \mathrm{s} . \mathrm{d}$.

The flat (ribbon) sources composed of a $\mathrm{La}_{2} \mathrm{O}_{3}$-epoxy mixture had effective widths of $76 \mu \mathrm{m}$ or $130 \mu \mathrm{m}$. The total activity after thermal neutron irradiation in the University of Michigan Ford Reactor for $80-100 \mathrm{~h}$ at a flux of approximately $2 \times 10^{13}$ $\mathrm{n} / \mathrm{cm}^{2} \cdot \mathrm{sec}$ was estimated to be 0.2 to $0.8 \mathrm{Cur}$. The sources were aligned in the spectrometer using the second-order reflection from the $\operatorname{Ge}(022)$ planes of the $487 \mathrm{keV}$ gamma ray. The resolution widths ( $\mathrm{fwhm}$ ) of the diffraction profiles obtained with the $\mathrm{Ge}(022)$ planes were approximately $10 \mathrm{sec}$ and $13.5 \mathrm{sec}$ of arc for the two different source widths. These correspond to an energy resolution of $\Delta E=16 \times 10^{-6} E^{2} / \mathrm{n}$ $\mathrm{keV}$ and $\Delta E=20 \times 10^{-6} E^{2} / n \mathrm{keV}$, respectively, where $E$ is in $\mathrm{keV}$ and $n$ the order of reflection. The resolution width obtained with the $Q(310)$ planes is 25 to $30 \mathrm{sec}$ of arc for both source widths. Data were taken using the first-through-fifth order reflection from the $\mathrm{Ge}(022)$ planes and the first-order reflection from the $Q(310)$ planes.

\subsection{LITHIUM-DRIFTED GERMANIUM SPECTROMETER}

The $\mathrm{Ge}(\mathrm{Li})$ spectrometer consists of the following components: a $\mathrm{Ge}(\mathrm{Li})$ detector having a sensitive volume of $0.2 \mathrm{~cm} \times 0.8 \mathrm{~cm}^{2}$, a Tennelec $100 \mathrm{C}$ pre-amplifier and TC 200 amplifier and a Victoreen (SCIPP) 1600-channel pulse-height analyser. The detector is operated at liquid nitrogen temperature. Typical resolution widths (fwhm) obtained for three lines in the decay of ${ }^{140} \mathrm{La}$ were $4.2 \mathrm{keV}$ for the $487 \mathrm{keV}$ line, 5.5 $\mathrm{keV}$ for the $1597 \mathrm{keV}$ line and $9.0 \mathrm{keV}$ for the $2522 \mathrm{keV}$ line. The data accumulation time varied from 30 to $2500 \mathrm{~min}$. It was possible to obtain the energy calibration from lines within the spectrum since energy measurements had been made with good accuracy using the ccs. The calibration was obtained from the three lines having energies $487.029 \pm 0.019,816.801 \pm 0.086$ and $1596.58 \pm 0.30 \mathrm{keV}$.

The source consisted of several $\mathrm{mg}$ of $\mathrm{La}\left(\mathrm{NO}_{3}\right)_{3}$ dissolved in water and irradiated with thermal neutrons for $2-4 \mathrm{~h}$ in the University of Michigan Reactor. The activity was about $1 \mathrm{mCur}$.

\section{Experimental Results}

\subsection{THE GAMMA-RAY SPECTRUM}

The gamma-ray energies measured in this study are listed in table 1. All energies 
were measured at least twice and in most cases three or four times. The uncertainties correspond to the standard deviation of the mean.

The relative intensities of some of the observed gamma rays are given in table 2 . In column two of this table are listed the relative intensities obtained from the present work. The intensity values in this column are separated into three subgroups and within each group the intensities are normalized to the most intense member of that group. In column four are listed the relative intensities reported in ref. " 1 ). All intensities in this column are relative to the $1597 \mathrm{keV} \gamma$-ray which was taken to have a

TABLE: 1

Energy values for the gamma rays in the "

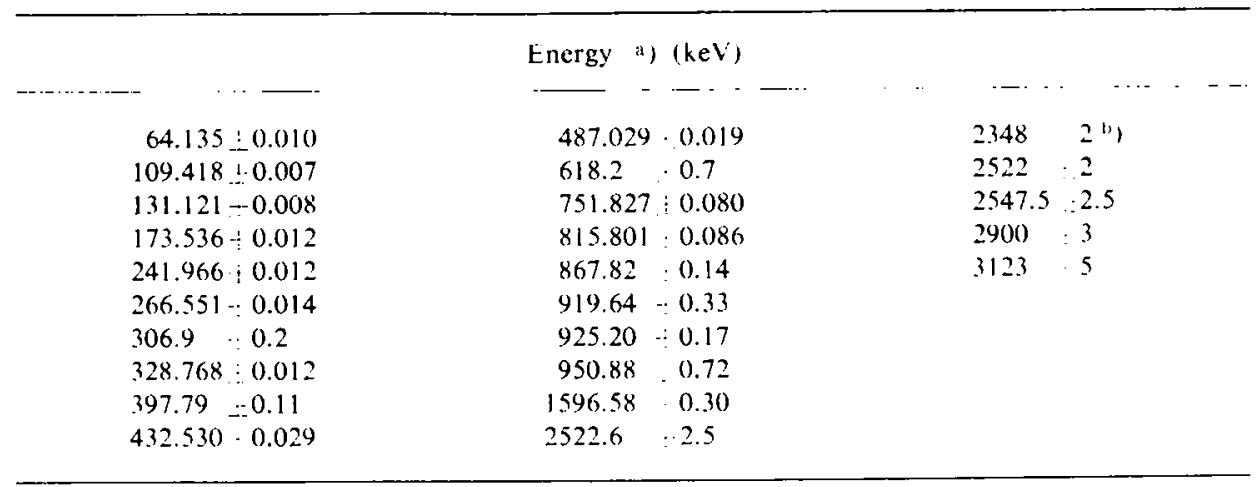

a) All values are derived from data obtained with a $2 \mathrm{~m}$ curved-crystal spectrometer. except where noted.

1) Values in this column derive from data ubtained with the Ge(Li) spectrometer.

value 1.00 . The numbers in this column closely correspond to the values for quanta; decay reported by Dzhelepov et al. ${ }^{4}$ ) when multiplied by 0.96 . In column five the relative intensities of ref. ${ }^{11}$ ) are renormalized to the scale of column two.

The relative intensities measured in the present work and listed in the first two subgroups were obtained with the ces using the Ge(022) planes in second-order reflection. The reflection efficiency of this crystal in second order, $R\left(E_{i}, 2\right)$, has been investigated ${ }^{10}$ ) in the energy range from 130 to $2110 \mathrm{keV}$ and is reasonably described by $R\left(E_{i}, 2\right) \times E_{i}^{-k}$ with $0.6 \leqq k \leqq 1.2$. A value of $k=1.0$ was used for the present work. The relative intensities were obtained from the relative peak counting rates corrected for crystal reflection efficiency and photopeak detection efficiency. The uncertainties in intensity values (column three) are derived from the uncertainty in the crystal reflection efficiency and the statistical fluctuations in the peak counting rates. The relative intensities of the third subgroup derive from the data taken with the $\mathrm{Ge}(\mathrm{Li})$ spectrometer. The ratio of full-energy peak amplitudes corrected for detection efficiency was used. The correction for detection efficiency was based on the relative photoelectric cross sections. 
The following energy measurements are of special interest:

TABLE 2

The relative intensities of some $\gamma$-rays in the ${ }^{140} \mathrm{La} \rightarrow{ }^{140} \mathrm{Ce}$ decay

\begin{tabular}{|c|c|c|c|c|c|c|}
\hline & $\begin{array}{c}\text { Gamma-ray energy } \\
\text { present work } \\
(\mathrm{keV})\end{array}$ & $\begin{array}{l}\left.I_{\gamma}{ }^{a}\right) \\
\text { present }\end{array}$ & work $^{\Delta I_{\gamma}}$ & $\begin{array}{c}I_{y}, \mathrm{l} \\
\text { Prikhodtseva and }\end{array}$ & $\begin{array}{l}\left.I_{y^{c}}\right) \\
\left.\text { Khol'nov }{ }^{11}\right)\end{array}$ & \\
\hline & 306.9 & 0.044 & 0.023 & & & \\
\hline & 328.768 & 45 & 10 & 0.21 & 50 & \\
\hline & 397.79 & 0.11 & 0.050 & 0.03 & 7 & \\
\hline & 432.530 & 6.1 & 1.5 & 0.025 & 6.0 & \\
\hline & 487.029 & 100 & & 0.419 & 100 & \\
\hline & 618.2 & 0.090 & 0.045 & & & \\
\hline & 751.826 & 19 & 3.8 & $\left(0.0337^{\mathrm{d}}\right)$ & 17.2 & \\
\hline & 815.801 & 100 & & 0.196 & 100 & \\
\hline & 867.82 & 22.5 & 4.5 & 0.053 & 27 & \\
\hline & 919.64 & 11 & 2.7 & & & \\
\hline & 925.20 & 30 & 6.0 & $\left.0.096^{e}\right)$ & 49 & \\
\hline & 950.88 & 2.6 & 0.65 & & & \\
\hline & 2348 & 33 & 9.0 & 0.0078 & 21 & \\
\hline & 2522 & 100 & & 0.037 & 100 & \\
\hline & 2547.5 & 3.5 & 1.0 & & & \\
\hline
\end{tabular}

a) The intensities of the present work are given relative to the most intense member within cach subgroup.

i) The values in this column are reported in ref. ${ }^{11}$ ) and correspond closely to the quantaidecay reported by Izhelepov et al. ") when multiplied by 0.96 .

c) The values of ref. ${ }^{11}$ ) renormalized to the scale of column two.

d) Intensity of a reported $748 \mathrm{keV}$ transition ${ }^{11}$ ).

e) Intensity of a reported $923 \mathrm{keV}$ transition ${ }^{11}$ ).

919.64 and $925.20 \mathrm{keV}$. Previously these two transitions have been reported as a single transition with an energy of approximately $923 \mathrm{keV}$. The diffraction profiles for this doublet obtained with two different source widths and dispersions are represented in fig. 1. Fig. 1(b) shows that these two lines are well separated in the fourth-order reflection from the $\mathrm{Ge}(022)$ planes using a source of $76 \mu \mathrm{m}$ width. The separation of the composite profile into two separate peaks was somewhat less in the $\mathrm{Ge}(\mathrm{Li})$ spectrum. Using the data of both instruments, the relative gamma-ray intensity value $I_{i}(919.64) / I_{j}(925.20)=0.37 \pm 0.04$ is obtained.

$950.88 \mathrm{keV}$. The diffraction profile for this previously unreported transition is shown in fig. la). The energy was determined using the second-order reflection from the $\mathrm{Ge}(0 \overline{2} 2)$ planes. The intensity of this gamma ray relative to the $920-925 \mathrm{keV}$ doublet was constant with time. On this basis the $950.88 \mathrm{keV}$ transition is assigned to the ${ }^{140} \mathrm{La} \rightarrow{ }^{140} \mathrm{Ce}$ decay.

$618.2 \mathrm{keV}$. The measurement on this weak transition had to be made in first-order reflection since the second-order diffraction profile contains a contribution of the relatively strong third-order reflection of the $920-925 \mathrm{keV}$ lines. The first-order diffraction profile of this gamma-ray line is shown in fig. $2 a$; in the same figure the 
$487 \mathrm{keV}$ diffraction profile is shown for comparison. Since the $487 \mathrm{keV}$ transition occurs in about $40 \%$ of the decays ${ }^{4}$ ), the $618.2 \mathrm{kcV}$ transition occurs only in approximately $4 \times 10^{-4}$ decays. It could not be seen in the singles spectrum obtained with the $\mathrm{Ge}(\mathrm{Li})$ spectrometer due to the presence of the Compton distributions of intense high-energy lines in the region of the $618.2 \mathrm{keV}$ photopeak.

$306.9 \mathrm{keV}$. This transition has not been reported previously in the decay of ${ }^{140} \mathrm{La}$ though it has been observed in the ${ }^{140} \mathrm{Pr} \rightarrow{ }^{140} \mathrm{Ce}$ decay ${ }^{5}$ ).

Although the $306.9 \mathrm{keV}$ transition is very weak the measurcment on this line necessitated using the second-order reflection from the $\mathrm{Ge}(022)$ planes for the fol-

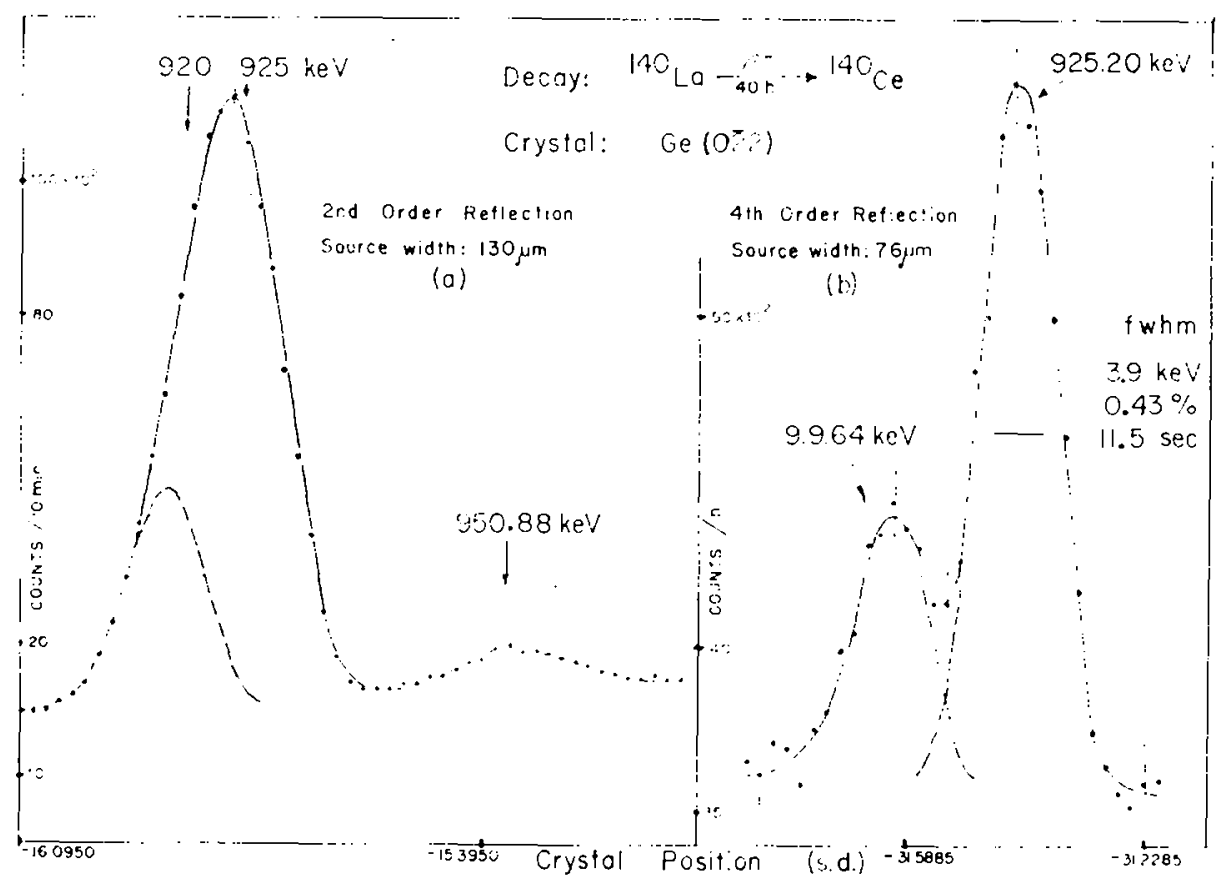

Fig. 1. Diffraction profiles of the $920-925 \mathrm{keV}$ gamma-ray doublet occurring in the decay of ${ }^{1+1} \mathrm{La}$ obtained with two different source widths and dispersions. The solid line merely indicates the trend of the data points.

lowing reason. The first-order reflection of the $306.9 \mathrm{keV}$ gamma ray occurs at nearly the same Bragg angle as the third-order reflection of the $920-925 \mathrm{keV}$ doublet. Thus the diffraction spectrum consists of three different gamma rays in various proportions of intensity. By setting the amplifier-discriminator to accept the photopcak of the $306.9 \mathrm{keV}$ gamma ray, a portion of the Compton distribution due to the 920-925 $\mathrm{keV}$ gamma rays is also accepted. In the region of the first-order reflection of the $306.9 \mathrm{keV}$ line (and so the third-order reflection of the $920-925 \mathrm{keV}$ doublet) the relative intensities of these two contributions to the count rate is large enough to produce serious distortions to the diffraction profile of the $306.9 \mathrm{keV}$ line. By measur- 
ing the $306.9 \mathrm{keV}$ gamma ray in second order this distortion is greatly reduced since the diffraction profile intensity of the $920-925 \mathrm{keV}$ lines (sixth-order reflection now) is reduced by about a factor of ten, whereas the $306.9 \mathrm{keV}$ line is only reduced in intensity by about a factor of two. In this manner a good energy determination of

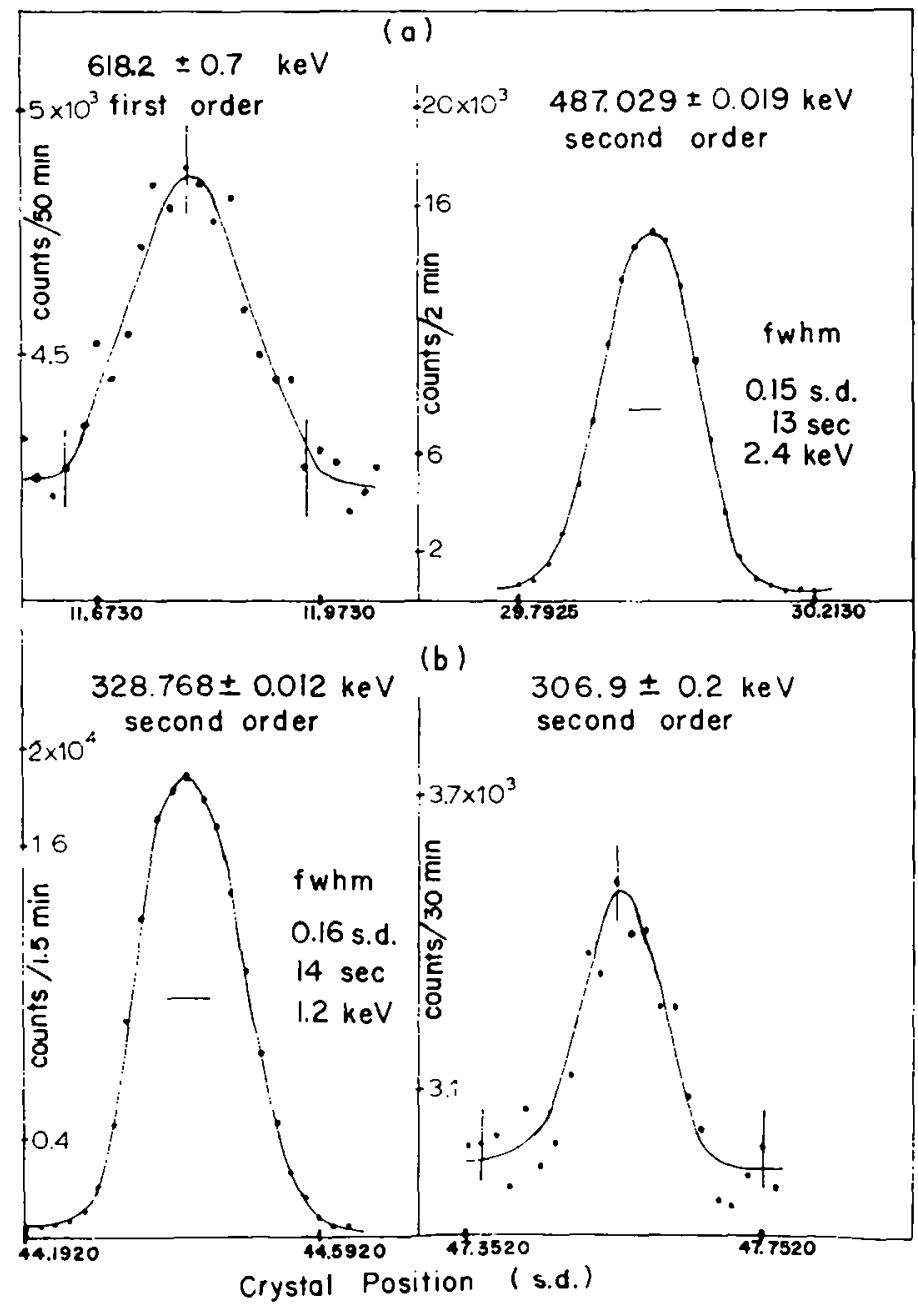

Fig. 2. Diffraction profiles of the weak 306.9 and $618.2 \mathrm{keV}$ gamma rays occurring in the decay of ${ }^{140} \mathrm{La}$. The profiles of the intense 487 and $329 \mathrm{keV}$ lines are shown for comparison. The crystal was $\mathrm{Ge}(0 \overline{2} 2)$ and the source activity was about $0.8 \mathrm{Cur}$.

this transition was possible, though it occurs only in approximately $2 \times 10^{-4}$ of the decays. Fig. 2b shows the second order diffraction profile of the $306.9 \mathrm{keV}$ line; the diffraction profile for $328.768 \mathrm{keV}$ line is shown for comparison. The $306.9 \mathrm{keV}$ line could not be observed in the singles spectrum obtained with the Ge(Li) spectrometer. 
2348,2522 and $2547.5 \mathrm{keV}$. The photopeaks of these three gamma rays in the $\mathrm{Ge}(\mathrm{Li})$ spectrum are shown in fig. 3. The $2547.5 \mathrm{kcV}$ gamma ray has not been reported previously. The positions of the photopeaks and double escape peaks were both used to determine the energies. The relative intensities of these three lines in the Ge(Li) spectrum stayed constant in time and thus were attributed to the decay of ${ }^{140} \mathrm{La}$.

$2900 \mathrm{keV}$. The best energy determination of this gamma ray could be made from the position of the double escape peak in the $\mathrm{Ge}(\mathrm{Li})$ spectrum. The photopeak was observed, but it was not used in the energy determination due to very low counting rates. The intensity of the double escape peak fell off with the same time constant as the $2522 \mathrm{keV}$ line.

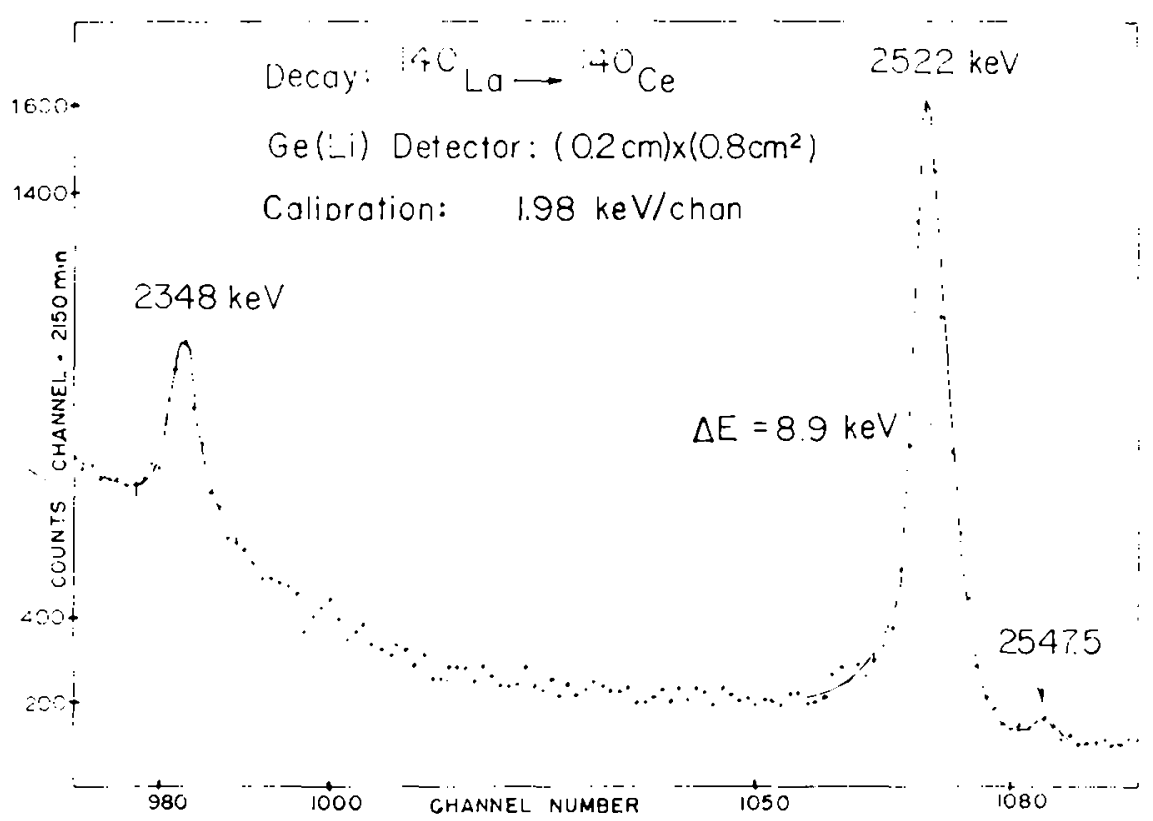

Fig. 3. The singles spectrum obtained with a $\mathrm{Ge}(\mathrm{Li})$ spectrometer in the energy region $2320-2570$ $\mathrm{keV}$ for the gamma rays which follow the decay of ${ }^{1+0} \mathrm{La}$.

$3123 \mathrm{keV}$. A good encrgy measurement of this previously reported gamma ray ${ }^{4}$ ) could not be made due to the low detection efficiency of the Ge( $\mathrm{Li}$ ) detector at this energy. However, the presence of a small peak at $2101 \pm 5 \mathrm{keV}$ could correspond to the double escape peak of a $3123 \mathrm{keV}$ gamma ray.

\section{Discussion of the Level Scheme for ${ }^{140} \mathrm{Ce}$}

In fig. 4 is presented a level scheme for the excited states of the nucleus ${ }^{140} \mathrm{Ce}$ populated through the beta decay of ${ }^{140} \mathrm{La}$ which is consistent with the present energy measurements and much of the work of previous investigators. To the level scheme 
of ref. ${ }^{3}$ ) two new levels at 2516.14 and $2547.5 \mathrm{keV}$ have been added and the tentatively proposed level at $2348.41 \mathrm{keV}$ is further supported. Angular momenta and parities were taken from ref. ${ }^{3}$ ), the compilation of data by Dzhelepov et al. ${ }^{4}$ ) and more recent angular correlation measurements ${ }^{5,7,8}$ ). The $\beta$-ray end-point energies were obtained using the value $Q_{\beta}=3.769 \pm 0.005 \mathrm{MeV}$ given by Mattauch et al. ${ }^{12}$ )

$$
\frac{140 \mathrm{Lo}}{T_{i_{2}}=40.23 \mathrm{~h}} 3^{-}
$$

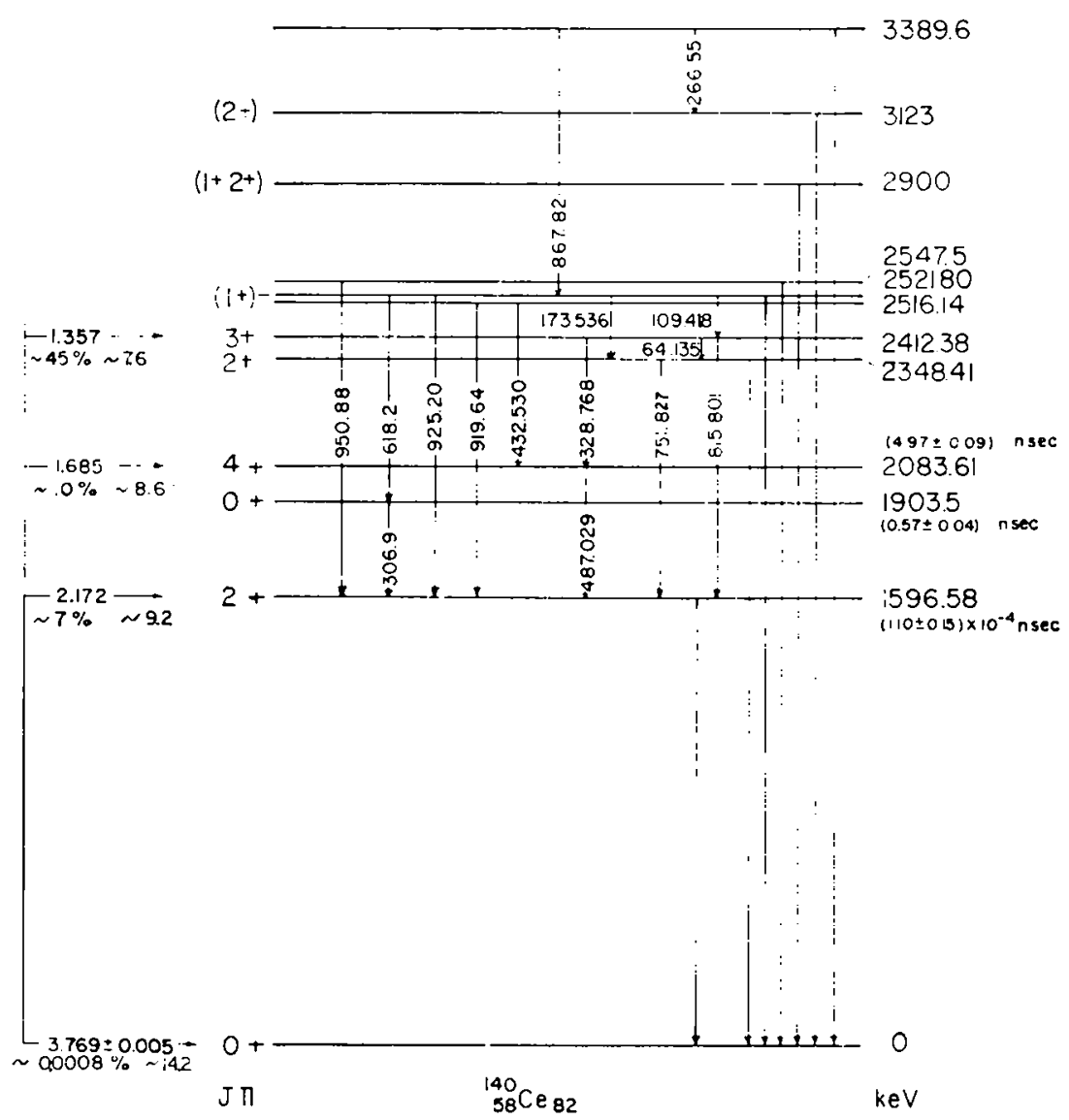

Fig. 4. The proposed level scheme for ${ }^{140} \mathrm{Ce}$ using the results of the present investigations.

and the level excitation energies from the present work. Only part of the $\beta$-decay fecding is shown since the softer $\beta$-ray components have not yet been clearly identified. The mean lives for the first three excited levels are taken from refs. ${ }^{16-18}$ ). The average value of the four measurements ${ }^{19}$ ) of the gyromagnetic ratio $g$ of the first $4^{+}$level is $1.07 \pm 0.05$. A discussion of some of the levels follows. 
$1903.5 \mathrm{keV}$. The absence of a $\gamma$-ray with this energy and the measurements on the conversion electrons and internal-pair positons emitted in the direct transition to the ground state led to a $J^{\pi}=0^{+}$assignment ${ }^{4}$ ) to this level. $\Lambda$ weak $0.63 \mathrm{MeV}$ $\gamma$-ray populating this level in the decay of ${ }^{140} \mathrm{La}$ has been reported ${ }^{6,21}$ ) and a $\gamma$-ray of energy $0.306 \mathrm{MeV}$ depopulating this level was found ${ }^{5}$ ) in the decay of ${ }^{140} \mathrm{Pr}$. On this basis the $618.2 \pm 0.7$ and $306.9 \pm 0.2 \mathrm{keV}$ transition measured in this work have been assigned to the ${ }^{140}$ La decay. Hisatake et al. ${ }^{5}$ ) have measured the $\gamma$-ray directional correlation for the $0.306-1.597 \mathrm{MeV}$ cascade occurring in the decay of ${ }^{140} \mathrm{Pr}$ and found it consistent with the $(0-2-0)$ angular momentum sequence.

$2348.41 \mathrm{keV}$. A level at this energy is established since a $\gamma$-ray of this energy has been observed in the spectrum. (Any transition in this decay with energy $\geqq 2.172$ $\mathrm{MeV}$ must be a ground state transition since $Q_{\beta}=3.769 \mathrm{MeV}$ and the first excited state is at $1.597 \mathrm{MeV}$.) The internal conversion electrons and internal-pair positons for a transition of this energy have also been observed ${ }^{4}$ ). Dzhelepov et al. ${ }^{4}$ ) have proposed a $J^{r}=2 *$ assignment for this level. In addition to the transitions which are shown in ref. ${ }^{3}$ ) to populate and depopulate this state it is proposed that the $64.135 \mathrm{keV}$ transition proceeds to this level from the $2412.4 \mathrm{keV}$ level. The measured energy $815.801 \pm 0.086 \mathrm{keV}$ of the crossover transition from the 2412.4 to the 1596.6 $\mathrm{keV}$ state compares favourably with the sum of the measured transition energies $751.827 \pm 64.135=815.962 \pm 0.081 \mathrm{keV}$.

$2412.38 \mathrm{keV}$. Many investigators ${ }^{7,17}$ ) have attributed a value $J^{\pi}=3^{+}$to this level on the basis of the 329-487, 816-1597, 329-1597 keV $\gamma$-ray directional correlation measurements and earlier electron conversion studies ${ }^{3}$ ). However, the quadrupole admixture $(0.1-0.5 \%)$ in the $329 \mathrm{keV}$ transition which is obtained from the $\gamma \gamma(\gamma)$ data ${ }^{19}$ ) does not agree with the admixtures which Bashilov et al. ${ }^{20}$ ) reported based on a comparison of the experimental value of $x_{K}$ and $K / L$ with the theoretical values of Sliv and Band. This apparent discrepancy can be partially resolved if instead one uses the revised table of theoretical conversion coefficients of Sliv and Band ${ }^{22}$ ) from which the following values of $x_{K}$ and $K / L$ are obtained for the different types of transitions (at $329 \mathrm{keV}$ ):

\begin{tabular}{lcccccc} 
& E1 & E2 & E3 & M1 & M2 & M3 \\
$\alpha_{K}\left(\times 10^{3}\right)$ & 8.5 & 30 & 92 & 38 & 155 & 530 \\
\hline $\mathrm{K} / \mathrm{L}$ & 7.45 & 5.3 & 2.8 & 7.1 & 6.3 & 4.8
\end{tabular}

The experimental value $\alpha_{\mathrm{K}}=29 \times 10^{-3}$ reported by Bashilov et al. still seems most consistent with the theoretical values for an E2 transition, though an appreciable Ml admixture might not be excluded (no uncertainties were reported in ref. ${ }^{20}$ )). The earlier theoretical value ${ }^{20}$ ) for $x_{K}(M 1)$ was $47 \times 10^{-3}$. This appeared to have eliminated the possibility of an appreciable $M 1$ admixture in the $329 \mathrm{keV}$ transition. Using the relative $\gamma$-ray intensity of the present work, $I_{\gamma}(329) / I_{\gamma}(487)=0.45 \pm 0.10$, the K-conversion relative electron intensity of Bashilov et al., $I_{\mathrm{Ke}}(329) / I_{\mathrm{Ke}}(487)=1.6$ 
and the theoretical value $x_{K}(487 \mathrm{keV} ; \mathrm{E} 2)=9.5 \times 10^{-3}$, the value $\alpha_{K}(329 \mathrm{keV})=$ $(34 \pm 7) \times 10^{-3}$ is obtained. This value is consistent with an $\mathrm{Ml}+\mathrm{E} 2$ transition, though the admixture cannot be specified. The experimental value ${ }^{20}$ ) $\mathrm{K} / \mathrm{L}=$ $6.2 \pm 0.5$ is also consistent with the new theoretical value for a $\mathrm{M} 1+\mathrm{E} 2$ transition. Since the $329 \mathrm{keV}$ transition proceeds to the $2083.6 \mathrm{keV}\left(2^{+}\right)$state, the assignment $J^{\pi}=3^{+}$is not excluded by conversion electron data and is in fact most consistent with all available data.

$2516.14 \mathrm{keV}$. A level with this excitation energy is proposed for the following two reasons. The measured $\gamma$-ray energy $919.64 \pm 0.33 \mathrm{keV}$ closely corresponds to the energy sum $487.029+432.530=919.559 \pm 0.035 \mathrm{keV}$. Bolotin et al. ${ }^{23}$ ) have reported coincidences between $\gamma$-rays with energies of approximately 487 and $433 \mathrm{keV}$. Six transitions from the $2516.14 \mathrm{keV}$ level to lower levels are possible and a search was made for some of the corresponding $\gamma$-rays. The energies of these transitions and the intensity limits determined for the unobserved $\gamma$-rays are as follows:

$$
\begin{array}{lllll} 
& \text { transition } & & \multicolumn{1}{r}{\text { relative intensity limits }} \\
J^{\pi} \rightarrow 3^{+} & 2516.14-2412.38=103.76 \mathrm{keV}, & I_{\gamma}(104 \mathrm{keV})<0.03 I_{\gamma}(131 \mathrm{keV}), \\
J^{\pi} \rightarrow 2^{+} & 2516.14-2348.41=167.71, & I_{\gamma}(168)<1.5 \times 10^{-3} I_{\gamma}(432.5), \\
J^{\pi} \rightarrow 4^{+} & 2516.14-2083.61=432.53, & & \text { measured }(\text { table } 2) \\
J^{\pi} \rightarrow 0^{+} & 2516.14-1903.5 & =612.6, & & I_{\gamma}(613)<5 \times 10^{-3} I_{\gamma}(432.5), \\
J^{\pi} \rightarrow 2^{+} & 2516.14-1596.58=919.56, & & \text { measured (table 2), } \\
J^{\pi} \rightarrow 0^{+} & 2516.14-\quad 0 & =2516.14, & & I_{\gamma}(2516)<0.1 I_{\gamma}(2522) .
\end{array}
$$

The data available for a $J^{\pi}$ assignment to this new level are the values $\alpha_{K}(432.5)=$ $(10 \pm 4) \times 10^{-3}$ and the relative intensity $I_{\gamma}(432.5) / I_{\gamma}(919.6)=1.2 \pm 0.4$. The value for $x_{\mathrm{K}}$ was obtained using the relative $\mathrm{K}$-conversion intensities $I_{\mathrm{eK}}(432.5) / I_{\mathrm{eK}}(487)=$ $0.06 \pm 0.016$ of Bashilov et al. (the uncertainty in this number is our estimate), the relative $\gamma$-ray intensity of the present work $I_{\gamma}(432) / I_{\gamma}(487)=0.060 \pm 0.015$ and the theoretical value $x_{\mathrm{K}}(487 ; \mathrm{E} 2)=9.5 \times 10^{-3}$. The relative $\gamma$-ray intensity was obtained using the value $I_{\gamma}(487) / I_{\gamma}(816)=2.14$ of $\left.\mathrm{ref} .{ }^{11}\right)$ and the values $I_{\gamma}(432.5) / I_{\gamma}(487)=$ $0.061 \pm 0.015$ and $I_{. j}(919.6) / I_{y}(815.8)=0.11 \pm 0.027$ of the present work. The theoretical values ${ }^{22}$ ) of $\alpha_{K}(432.5)$ for the different types of transitions are:

$\begin{array}{rrrrlllc}\alpha_{K}\left(\times 10^{3}\right) & \text { E1 } & \text { E2 } & \text { E3 } & \text { M1 } & \text { M2 } & \text { M3 } & \text { experiment } \\ & 4.4 & 13.2 & 37 & 19 & 64 & 195 & 10 \pm 4 .\end{array}$

Thus the experimental value is compatible with the theoretical values for transitions of type E1+M2, E2 + M1 and pure E2. Since the $432.5 \mathrm{keV}$ transition populates a $4^{+}$state, the values of $J^{\pi}$ for the $2516 \mathrm{keV}$ level may be $3^{-}, 4^{-}, 5^{-}$or $2^{+}, 3^{+}, 4^{-}, 5^{+}$. Since the ${ }^{14(0)}$ La ground state has $J^{\pi}=3^{-}$, the apparent absence of $\beta$-ray feeding ${ }^{3.4}$ ) to the $2516 \mathrm{keV}$ level tends to exclude the negative parity states. The observed $\gamma$-ray intensity $I_{i}\left(432.5 ; J^{\pi} \rightarrow 4^{+}\right) / I_{y j}\left(919.6 ; J^{\pi} \rightarrow 2^{+}\right)=1.2 \pm 0.4$ tends to exclude the high angular momentum states $5^{+}, 6^{+}$. Thus the values $J^{\pi}=2^{+}, 3^{+}, 4^{+}$appear to be most compatible with the data. It might further be argued, that a $2^{+}$assignment is un- 
likely since the transition probability $\lambda(2516$; E2) should be much larger than the transition probability $\lambda(432.5$, E2), and yet the $2516 \mathrm{keV}$ transition is not observed. $\mathrm{A}^{+}$assignment would lead to the unusual circumstance of having two $3^{+}$levels separated by $103.8 \mathrm{keV}$ with one $(2412 \mathrm{keV})$ being fed in about $50 \%$ of the $\beta$-decays, ${ }^{3}$ ) the other fed very little (if at all). The assignment $J^{\pi}=4^{+}$to the $2516 \mathrm{keV}$ level does not seem to violate any of these elementary notions. On the basis of these weak arguments the value $J^{\pi}=4^{+}$is favoured.

$2521.80 \mathrm{keV}$. The $\gamma$-rays, electron conversion electrons and internal pair positons corresponding to the direct transition from this level to the ground state have been observed ${ }^{3,4}$ ). Heretofore the $J^{\pi}$ assignment ${ }^{3,4}$ ) has been $2^{+}$and this was based on data from the "923"- $1597 \mathrm{keV} \gamma$-ray directional correlation and electron conversion data on the 433 and " 923 " keV transitions. Now that the $433 \mathrm{keV}$ transition can no longer be considered to depopulate the $2522 \mathrm{keV}$ level and the " 923 " keV $y$-ray has been resolved into two components with energies 919.64 and $925.20 \mathrm{keV}$ with a relative intensity of $I_{\gamma}(919.6)=0.37 I_{7,}(925.2)$, the $J^{\pi}=2^{\prime}$ assignment must be reevaluated. The data available for a determination of $J^{\pi}$ for the $2522 \mathrm{keV}$ level are the measurements $\left.{ }^{13}\right) x_{K}(2522)=3.91 \times 10^{-4}$ and the ratio of internal-pair positons to $\mathrm{K}$-shell conversion electrons $\left.{ }^{15}\right) I_{\mathrm{c}^{+}} / I_{\mathrm{c}} \cdot=1.05$. Both of these measurements, when compared to the theoretical values ${ }^{13.15}$ ), indicate that the $2522 \mathrm{keV}$ transition to the ground state $\left(0^{+}\right)$is of type Ml. Thus the assignment $J^{\pi}=1^{-}$to the $2522 \mathrm{keV}$ level is most likely. Also, the measured value ${ }^{20}$ ) of $\mathrm{K} / \mathrm{L}$ for the $109.42 \mathrm{keV}$ transition from the $2522 \mathrm{keV}$ to the $2412 \mathrm{keV}\left(3^{\prime}\right)$ level is consistent with the theoretical value for a pure E2 transition. This measurement is therefore also compatible with a 1 ' assignment to the $2522 \mathrm{keV}$ state.

It is seen from the level scheme (fig. 4) that the $\gamma$-rays for five out of the seven possible transitions depopulating the $2522 \mathrm{keV}$ level have been observed. The $\gamma$-ray of the transition going to the $2084 \mathrm{kcV}\left(4^{+}\right)$level was not observed. The intensity limit $I_{\gamma}(438.14)<0.03 I_{i}(432.5)$ was determined.

$2547.5 \mathrm{keV}$. A new level is proposed at this excitation energy because a ;-ray of this energy is observed in the spectrum. Further, the previously unreported $\gamma$-ray with an energy of $950.88 \mathrm{keV}$ could correspond to the transition from this level to the first excited state at $1596.58 \mathrm{keV}$. The sum $1596.58+950.88=2547.5 \pm 0.77$ $\mathrm{keV}$ agrees well with the measured encrgy $2547.5 \pm 2.5 \mathrm{keV}$. There are no data available for a direct determination of $J^{\pi}$ for this level. Some weak arguments based on the observed $\gamma$-ray intensities favour the values $J=1,2$.

A search was made for a $199 \mathrm{keV} \gamma$-raly corresponding to the transition from the $2547.5 \mathrm{keV}$ to the $2348.4 \mathrm{kcV}$ level. With the curved crystal spectrometer, a peak could be seen at the crystal setting for the first-order reflection $(\mathrm{Ge}(022))$ of this line. However, the second-order reflection of the $397.79 \mathrm{kcV} \gamma$-ray occurs at nearly the same Bragg angle and therefore could account for the observed peak. Unfortunately, the available intensity was insufficient to go to a higher order reflection where an 
identification of the peak would be possible. Nevertheless, the intensity limit $I_{\nu}$ $(199 \mathrm{keV})<1.9 \times 10^{-4} I_{\gamma}(487 \mathrm{keV})$ could be set.

2900,3123 and $3389.6 \mathrm{keV}$. These levels are included in the decay scheme since the direct transitions to the ground state have been observed ${ }^{4}$ ). Also there is at least one soft component in the $\beta$-ray spectrum with a reported ${ }^{3,4}$ ) end-point energy of $0.42 \mathrm{MeV}$ which could feed into one of the upper levels. The $J^{\pi}$ values for these levels shown on the level scheme are those given by Dzhelepov et al. ${ }^{4}$ ). In this investigation the 2900 and $3123 \mathrm{keV} \gamma$-rays were observed. A search was made for various $\gamma$-rays which would be emitted when these levels depopulate to lower-lying levels; however none was observed. Intensity limits could be set as follows: for $\gamma$ rays corresponding to the transitions $2900 \rightarrow 2348,1597 \mathrm{keV}$ the limits are $I_{\gamma}(566-534 \mathrm{keV})<3 \times 10^{-4} I_{\gamma}(487 \mathrm{keV})$ and $I_{\gamma}(1263-1345 \mathrm{keV})<3 \times 10^{-3} I_{\gamma}$ $(1597 \mathrm{keV})$; for $\gamma$-rays corresponding to the transitions $3123 \rightarrow 2348,1597 \mathrm{keV}$, the limits are $I_{\gamma}(788-772 \mathrm{keV})<3 \times 10^{-2} I_{\gamma}(751.8 \mathrm{keV})$ and $I_{\gamma}(1560-1500 \mathrm{keV})<1 \times 10^{-2}$ $I_{\gamma}(1597)$. The $\gamma$-ray for a previously reported $730 \mathrm{keV}$ transition ${ }^{20}$ ) could not be found and the intensity limit $I_{\gamma}(743-718 \mathrm{keV})<5 \times 10^{-2} I_{\gamma}(751.8 \mathrm{keV})$ was determined.

The moderately strong $867.82 \mathrm{keV} \gamma$-ray could correspond to the transition between the 3389.6 and $2521.80 \mathrm{keV}$ levels. This would account for the extensive depopulation of the $2521.8 \mathrm{keV}$ level. From the data on the $\beta$-ray spectrum it is not clear to what extent (if any) the $2521.80 \mathrm{keV}$ level is fed. The soft component in the $\beta$-ray spectrum with an end-point energy $0.42 \mathrm{MeV}$ could be feeding the $3389.6 \mathrm{keV}$ level. The observed $266.551 \mathrm{keV} \gamma$-ray could correspond to the transition from the $3389.6 \mathrm{keV}$ to the $3123 \mathrm{keV}$ level since the two sums $3123+266.5=$ $3189.5 \pm 5 \mathrm{keV}$ and $867.82+2521.80=3389.6 \pm 0.4 \mathrm{keV}$ are in good agreement.

It could not be determined where the $\gamma$-rays with energies $397.79,131.121$ and $241.966 \mathrm{keV}$ should be placed in the proposed level scheme. The measured energy $397.79 \pm 0.11 \mathrm{keV}$ agrees well with the sum $131.121 \pm 266.551=397.672 \pm 0.014$ $\mathrm{keV}$, suggesting that there may be a level at $3123-131=2992 \mathrm{keV}$ or at $3389.6+131.1$ $=3520.7 \mathrm{keV}$. However, no further support for the existence of such levels was found.

An application of the quasi-particle theory for single-closed shell nuclei (in BCS plus Tamm-Dancoff approximation) has recently been made by Rho ${ }^{2}$ ) to calculate the level spectra for $J^{\pi}=2^{+}, 3^{+}, 4^{+}$states of some spherical even-mass nuclei with a closed neutron shell at $N=82$. The nucleus ${ }^{140} \mathrm{Ce}$ was of special interest. In ${ }^{140} \mathrm{Ce}$ the two quasi-particle states having mostly

$$
\left(\mathrm{g}^{2} \frac{7}{2}\right)_{J=2}, \quad\left(\mathrm{~g}^{2} \frac{7}{2}\right)_{J=4}, \quad\left(\mathrm{~d}^{2} \frac{5}{2}\right)_{J=2}, \quad\left(\mathrm{~d}^{2} \frac{5}{2}\right)_{J=4}
$$

configurations are predicted to be the first four excited states respectively (ignoring $J=0,1$ states). In the presently proposed level scheme (fig. 4) these four states might be identified with the levels at excitation energies of $1.596\left(2^{+}\right), 2.084\left(4^{+}\right), 2.348$ $\left(2^{+}\right), 2.516\left(4^{+} ?\right) \mathrm{MeV}$ in the same order as above. The $J^{\pi}=3^{+}$level at $2.412 \mathrm{MeV}$ is likely the first of the $\left(\mathrm{d}_{2}^{5}, \mathrm{~g}_{2}^{7}\right)_{J}$ states and the $2.522 \mathrm{MeV}$ level may correspond to the $\left(\mathrm{d} \frac{5}{2}, \mathrm{~g} \frac{7}{2}\right)_{J=1}$ state. The theoretical value of Rho for the gyromagnetic ratio 
of the $2.084\left(4^{+}\right) \mathrm{keV}$ state is $g=0.92$ which compares favourably with the experimental value $g=1.07 \pm 0.05$ (average of the four measurements given in ref. ${ }^{19}$ )). This supports the hypothesis that the $2.084\left(4^{i}\right) \mathrm{keV}$ level has a predominantly $\left(\mathrm{g}_{2}^{2}\right)_{J=4}$ configuration. Further points of comparison are $B(\mathrm{E} 2)$ values. The measured $B\left(\mathrm{E} 2 ; 2^{+} \rightarrow 0^{+} ; 1597 \mathrm{keV}\right)$ and $B\left(\mathrm{E} 2 ; 4^{+} \rightarrow 2^{+} ; 487 \mathrm{keV}\right)$ are 16 and ${ }_{2}^{\frac{1}{8}}$ Weisskopf units, respectively, whereas the calculations predict 13 and $\frac{1}{17}$ Weisskopf units for the same quantities. In view of the experimental uncertainties, this agreement is good.

Although the comparison between theory and experiment that is at present available in the nucleus ${ }^{140} \mathrm{Ce}$ is modest, it should be mentioned that this nucleus has properties favourable to experimental and theoretical investigations which could with further investigation lead to a fruitful comparison.

The authors wish to thank A. B. Miller for his assistance in source preparation. One of us (H.B.) gratefully acknowledges the stimulating discussions with Professor K. T. Hecht and with his friend, I. P. Auer.

Note added in proof. The calculated (M. Rho, private communication) excitation energy of the two quasi-particle state $\left(g_{i}\right)_{J \pi=1}$. is approximately $2.5 \mathrm{MeV}$ and the $\left(\mathrm{g}_{2},{ }_{3}\right)_{J^{\prime}=3^{+}}$state is at a slightly lower energy. These states might correspond to the 2521.8 and $2412.4 \mathrm{keV}$ levels of the presently proposed level scheme.

\section{References}

1) I. S. Kisslinger and R. A. Sorensen, Mat. Fys. Medd. Dan. Vid. Selsk. 32, No. 9 (1960)

2) M. Rho, Congrès International de Physique Nucléaire, Vol. II, ed. by P. Gugenberger (Éditions du Centre National de la Recherche Scientifique, Paris, 1964) p. 440; M. Rho, Nuclcar Physics 65 (1965) 497

3) Nuclear Data Sheets, National Academy of Sciences, National Research Council, Wash. 25, D.C.

4) B. S. Dzhelepov, V. P. Prikhodtseva and Yu. V. Khol'nov, Isobaric nuclei with the mass number A - 140 (Pergamon Press, New York, 1963)

5) K. Hisatake, Y. Yoshida, K. Etoh and T. Murata, Nuclear Physics 56 (1964) 625

6) H. Takckoshi, N. Shikazono and P. Tseng, J. Phys. Soc. Japan 16 (1961) 1674

7) L. Dorikens-Vanpraet, O. Segacrt, M. Dorikens and J. Demuynck, Proc. Phys. Soc. 82 (1963)488

8) W. W. Black and A. C. G. Mitchell, Phys. Rev. 132 (1963) 1193

9) J. J. Reidy and M. 1.. Wicdenbeck, Nucl. Instr. 33 (1965) 21.3

10) J. J. Reidy and M. L. Wiedenbeck, Nuclear Physics 70 (1965) 518

11 ) V. P. Prikhodtseva and Iu. V. Khol'nov, Izv. Akad. Nauk SSSR (ser. fiz) 22 (1958) 176

12) J. H. E. Mattauch, W. Thicle and A. H. Wapstra, Nuclear Physics 67 (1965) I

13) B. S. Dzhelepov, Yu. V. Khol'nov and V. P. Prikhodtseva, Nuclear Physics 9 (1958) 665

14) V. A. Balalaev. B. S. Dthelepov, A. I. Medvedev. A. Meshter and I. F. Echevatkin. JEIP (Soviel Phys). 16 (1963) 1425

15) S. F. Antonova, S. S. Vasilenko, M. G. Kaganskii and D. L. Kaminskii, JETP (Soviet Phys.) 11 (1960) 554

16) S. Ofer and A. Schwarzschild, Phys. Rev. 116 (1959) 725

17) W. M. Curric, Nuclear Physics. 32 (1962) 574; 48 (1963) 561

18) R. L. Graham and J. S. Geiger. Bull. Am. Phys. Soc. 11 (1966) II

19) M. Schmorak, H. Wilson. P. Gatti and L. Grodzins, Phys. Rev. 134 (1964) B718

20) A. A. Bashilov, B. S. Dzhelepov, N. D. Novosil'tseva and L. S. Chervinskaya, Izv. Akad. Nauk SSSR (ser. fiz) 22 (1958) 179

21) P. Salling, Nuclear Physics 65 (1965) 520

$22)$ L. A. Sliv and I. M. Band, in Alpha-, beta- and gamina-ray spectroscopy, Vol. II, ed. by $K$. Siegbahn (North-Holland Publ. Co.. Amsterdam, 1965) appendix 5, p. 1639

23 ) H. H. Bolotin, C. H. Pruett, P. I. Roggenkamp and R. G. Wilkinson, Phys. Rev. 99 (1955) 62 